\title{
Evidence for Condon domains in white tin with two de Haas-van Alphen periods
}

\author{
G. Solt, ${ }^{1}$ V. S. Egorov, ${ }^{2}$ C. Baines, ${ }^{1}$ D. Herlach, ${ }^{1}$ and U. Zimmermann ${ }^{1}$ \\ ${ }^{1}$ Paul Scherrer Institut, CH-5232 Villigen PSI, Switzerland \\ ${ }^{2}$ Russian Research Center “Kurchatov Institute," Moscow 123182, Russia \\ (Received 9 June 2000)
}

\begin{abstract}
The observation of Condon domains in white tin, obtained by muon spin rotation spectroscopy, is reported. The experiment at $T=0.08 \mathrm{~K}$ for external magnetic fields between 1 and $2.6 \mathrm{~T}$ perpendicular to the (100) crystal plane reveals two "active" extremal cross sections of the Fermi surface, leading to domain formation with two different de Haas-van Alphen frequencies $F\left(\tau_{2}^{1}\right)$ and $F\left(\pi_{2}^{1}\right)$ at different field ranges and having a crossover region with no periodicity.
\end{abstract}

The periodic phase transitions generating dia- and paramagnetic domains in nonmagnetic $(s p)$ metals in a homogeneous magnetic field $\boldsymbol{H}$, predicted by Condon, ${ }^{1}$ arise because of the instability of the spatially uniform state of the electron system against "too large", de Haas-van Alphen (dHvA) oscillations. This is the case for

$$
\chi_{a}(B, T)=(\partial M / \partial B)_{\max }>1 / 4 \pi
$$

(where $\chi_{a}$ is the amplitude of the oscillating susceptibility $\chi=\partial M / \partial B$ and $B, M$ are components along $\boldsymbol{H}$ ), implying that the induction $B$ as a function of $H$ is multivalued. For a rod oriented parallel to the field, a discontinuous change of $B$ from a lower $\left(B_{1}\right)$ to a higher $\left(B_{2}\right)$ value takes place in each $d H v A$ period $^{1,2}$ with a jump of the magnetization from a diamagnetic $\left[M\left(B_{1}\right) \equiv M_{d}<0\right]$ to a paramagnetic $\left[M\left(B_{2}\right)\right.$ $\left.\equiv M_{p}>0\right]$ state, the ascending $\chi>0$ sections of the cycles are not realized. For a platelike sample set normal to $\boldsymbol{H}$, the discontinuity of $B$ and the requirement of flux conservation lead, for $B_{1}<H<B_{2}$, to the break-up of the uniform state and the coexistence of alternating oppositely magnetized regions with $B=B_{1}$ and $B=B_{2}$, the relative volumes of the dia- and paramagnetic domains adjusting themselves to satisfy $\bar{B}=H$.

In the limit $T, T_{D} \rightarrow 0 \quad\left(T_{D}\right.$ is the Dingle temperature) the crossing of the edge of a Landau subband with the Fermi level can be interpreted as an electronic topological transition $(\mathrm{ETT})^{3,4}$ at a Lifshitz-type singularity. Since this ETT would, however, be associated with an infinite susceptibility ${ }^{2}$ corresponding, by Eq. (1), to an unstable region, it is preceded (and replaced) by a first-order ETT leading to the Condon domain state. For a quasi-two-dimensional (2D) band structure the chemical potential is pinned ${ }^{5}$ in both the paraand diamagnetic domains at equally high, exactly filled Landau levels [the $n$th and $(n+1)$ th, respectively], and zero resistivity within each domain has been predicted. In normal metals with overlapping Landau subbands the situation is more complex, the subject keeps attracting intense theoretical interest. ${ }^{3,6-8}$

Up to now direct, spectroscopic evidence for Condon domains was obtained only in two cases: for silver, by NMR, ${ }^{9}$ and recently for $\mathrm{Be}$ by muon spin rotation ( $\mu \mathrm{SR})$ spectroscopy. ${ }^{10,11}$ Yet this scarcity of data does not mean that Condon domains are "exotic," it is due to experimental dif- ficulties (a need for highly perfect single crystals, detecting small field splittings $\Delta B=B_{2}-B_{1} \approx 10-50 \mathrm{G}$ in applied fields of some $T$, at temperatures $\lesssim 1 \mathrm{~K}$ ). In the "normal", 3D case (when the Lifshitz-Kosevich formula ${ }^{2}$ applies), Eq. (1) for the first harmonic of a given dHvA mode $i$ has the form

$$
\chi_{a}^{(i)}=\left(B_{0} / B\right)^{3 / 2} \exp \left(-B_{D} / B\right)\left(B_{T} / B\right) / \sinh \left(B_{T} / B\right)>1 / 4 \pi,
$$

where $B_{0} \equiv B_{0}^{(i)}$ is entirely determined by the Fermi surface (FS) parameters (cyclotron mass $m_{i}^{*}$, extremal crosssectional area $\mathcal{A}_{i}$ normal to $\boldsymbol{H}$, derivative $\mathcal{A}_{i}^{\prime \prime}=\partial^{2} \mathcal{A}_{i} / \partial d k_{z}^{2}$ along the field direction), $B_{D} \equiv B_{D}^{(i)}=\left(2 \pi^{2} k_{B} m_{i}^{*} c / \hbar e\right) T_{D}$ and $B_{T} \equiv B_{T}^{(i)}=\left(2 \pi^{2} k_{B} m_{i}^{*} c / \hbar e\right) T$. This inequality is always satisfied for some interval of $B$ provided $T_{D}$ and $T$ are sufficiently low. ${ }^{2,8,12}$ The results below show this in the case of metallic (white) tin.

Tin has several low lying dHvA frequencies $F_{i}$ $=(\hbar c / 2 \pi e) \mathcal{A}_{i}$ for which the dHvA period $\Delta H=H^{2} / F$ is sufficiently long even for moderate fields of $H<3 \mathrm{~T}$. This facilitates a fine mapping of the oscillatory cycle, which is necessary, since the "domain" section may be only a very small fraction of the dHvA period. This is certainly so for $4 \pi \chi_{a} \gtrsim 1$, but may be the case also for $4 \pi \chi_{a} \gg 1$ when both $T$ and $T_{D}$ are very low $\left(B_{T}, B_{D} \ll B\right)$, due to the cusps of the oscillating magnetization $M$ in this case ${ }^{2}$ even when its amplitude $M_{0}$ is much smaller than the period $\left(8 \pi^{2} M_{0}\right.$ $\ll \Delta H)$. The FS of white $\operatorname{tin}^{13-19}$ accommodates eight electrons, with electron sheets in the 4th, 5th, and 6th Brillouin zones (BZ). For $\boldsymbol{H} \|[100]$, one has five low-frequency dHvA oscillations, out of which three modes with the orbits $\tau_{2}^{1}, \pi_{2}^{1}$, and $\epsilon_{2}^{2}$ have sizeable amplitudes. ${ }^{17,18}$ The lowest frequency $F\left(\tau_{2}^{1}\right)=446.8 \pm 0.1 \mathrm{~T}$ (Ref. 19) is related to the central cross-sectional area of the "molar tooth" shaped FS sheet in the sixth zone, ${ }^{15}$ with cyclotron mass $m^{*} / m_{0}=0.29 \pm 0.06 .{ }^{16}$ Besides the small $m^{*}$, the low "longitudinal" curvature $\mathcal{A}^{\prime \prime} \approx 0.7$ at this cross section ${ }^{19}$ is also favorable for satisfying Eq. (2) $\left[B_{0} \propto 1 /\left(m^{* 2} \mathcal{A}^{\prime \prime}\right)^{1 / 3}\right.$ (Ref. 2)]. The mode $\pi_{2}^{1}$, with frequency $F\left(\pi_{2}^{1}\right)=2080 \pm 20 \mathrm{~T}$ and with $m^{*} / m_{0}$ between 0.55 and $0.79^{16,17}$ corresponds to the extremal cross section of the "pears and tubes" FS sheet in the fifth BZ, and the orbit $\epsilon_{2}^{2}\left[F\left(\epsilon_{2}^{2}\right)=3400 \pm 50 \mathrm{~T}\right]$ lies on the "earring" section in the fourth BZ. 


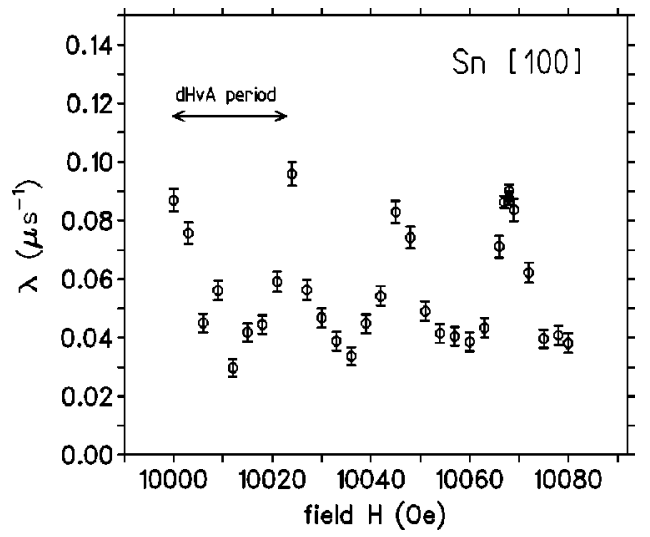

FIG. 1. Variation of the exponential damping rate $\lambda(H)$ (line broadening) for $T=0.08 \mathrm{~K}$ for white tin, $\boldsymbol{H} \|[100]$. The observed period $\Delta H \approx 23 \mathrm{G}$ agrees with the value of $22.6 \mathrm{G}$ corresponding to the dHvA frequency $F\left(\tau_{2}^{1}\right)=446.8 \mathrm{~T}$ (Ref. 19). The periodic peak regions are interpreted as due to the Condon domain state with oppositely magnetized regions, with $4 \pi \Delta M=\Delta B \approx \lambda / 2 \gamma_{\mu}$ $\approx 2.5 \mathrm{G}$.

In the experiment, an ultrahigh purity $\mathrm{Sn}$ crystal plate $\left[18 \times 12 \times 0.56 \mathrm{~mm}^{3}\right.$, electron mean free path $\approx$ some $\mathrm{mm}$ (Ref. 13)] grown parallel to the plane (100) was put into the field $\boldsymbol{H} \|[100]$ and cooled down to $T=0.08 \mathrm{~K}$. Transverse field $\mu \mathrm{SR}$ measurements, ${ }^{20}$ monitoring the time-dependent polarization [frequency(ies), damping rate(s), and amplitude(s)] of the precessing $\mu^{+}$spins were performed at the surface muon beamline $\pi \mathrm{M} 3$ of PSI, Villigen, as in Refs. 10 and 11 , by varying $H$ in small steps in different regions between 1 and $2.6 \mathrm{~T}$. The background noise was reduced by use of the MORE (muons-on-request) technique. ${ }^{21}$

Field scans were done first at $H=1 \mathrm{~T}$, where preliminary, low resolution data indicated an oscillating component in the linewidth. ${ }^{22}$ Mapping of a few adjacent dHvA cycles [with period $\Delta H \approx H^{2} / F\left(\tau_{2}^{1}\right) \approx 23 \mathrm{G}$ ] gives the result shown in Fig.1, where the damping rate $\lambda$ (line broadening in frequency space) is plotted against the applied field $H$. The sharp peaks are evidence of a periodic, sudden broadening of the field distribution in the bulk of the sample, which is difficult to reconcile with any single-phase state. [Should, for example, the variation of $\lambda$ reflect a spread $|\delta B|$, due to the nonellipsoidal shape of the sample and oscillating with $\chi$, the period for $\lambda$ would be that of $|\chi(B)|$, i.e., $\Delta H / 2$.] Moreover, the peaks in $\lambda$ are similar to those observed in $\mathrm{Be},{ }^{10,11}$ where the "broad" lines turned out to be doublets, with distinct frequencies for the dia- and paramagnetic domains. This suggests that the peaks in Fig. 1 are equally signs of the domain splitting, with the corresponding $\Delta B \approx 2 \lambda / \gamma_{\mu}$ $\approx 2.5 \mathrm{G}$ too small to allow resolution of the doublet. (Here $\gamma_{\mu}=2 \pi \times 13.554 \mathrm{kHzG}^{-1}$ is the gyromagnetic ratio of the muon; the experimental resolution was $\Delta B \approx 6-8$ G.) The next scans were done in successively higher fields, with the aim to increase $\Delta H$ and, eventually, also $\Delta B$. At $H$ $=1.41 \mathrm{~T}$, the oscillation of $\lambda$ looks the same as in Fig. 1 but with a period $\Delta H=46 \mathrm{G}$ twice as long, the peak values $\lambda$ $\approx 0.14 \mu \mathrm{s}^{-1}$ are still too small for an assumed doublet to be resolved.

By a further increase of the field at $H=2 \mathrm{~T}$, unexpectedly, a radically different picture seen in Fig. 2 was obtained:

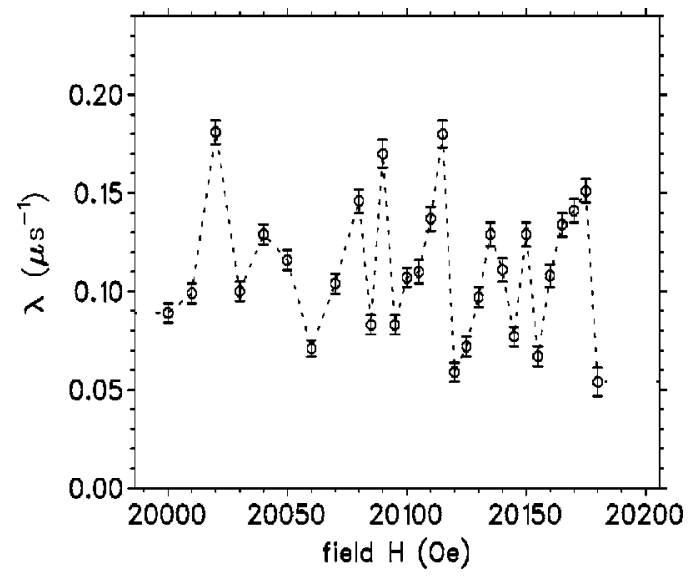

FIG. 2. Loss of periodicity in the damping rate $\lambda(H)$ near $H$ $=2 \mathrm{~T}$. The peaks mark, as before, the presence of highly inhomogeneous magnetization and possibly domains, but these cannot be related to any single dHvA mode.

the points for $\lambda(H)$ seem to be randomly scattered within the range of $0.1-0.2 \mu \mathrm{s}^{-1}$, showing no periodicity at all. Though the large peaks of $\lambda$ indicate, here also, the presence of highly inhomogeneous structures, the relation to quantum oscillations seems to be lost.

At the still higher field $H=2.6 \mathrm{~T}$, as seen in Fig. 3, the periodicity of $\lambda(H)$ reappears, but the period $\Delta H=33 \mathrm{G}$ shows that the "active" dHvA frequency has changed: instead of $F\left(\tau_{2}^{1}\right)$ as in Fig. 1 , one has $F=H^{2} / \Delta H=2064 \mathrm{~T}$. This agrees with the value $F\left(\pi_{2}^{1}\right)=2080 \pm 20$ T for the $\pi_{2}^{1}$ mode in the fifth $B Z$, for which the curvature of the FS along $\boldsymbol{H} \|[100]$ is also small, ${ }^{14}$ favorizing domain formation.

The appearance of the second "domain active" dHvA mode gives the explanation for the absence of periodicity near $H=2 \mathrm{~T}$. Equation (1) predicts periodic instabilities when a single mode, with amplitude $\chi_{a}^{(i)}>1 / 4 \pi$, is "dominant' in the given field range. The prevalence of one mode in certain field ranges is due to the fact that, by Eq. (2), $\chi_{a}^{(i)}(B)$ has a maximum for each mode, with the positions of

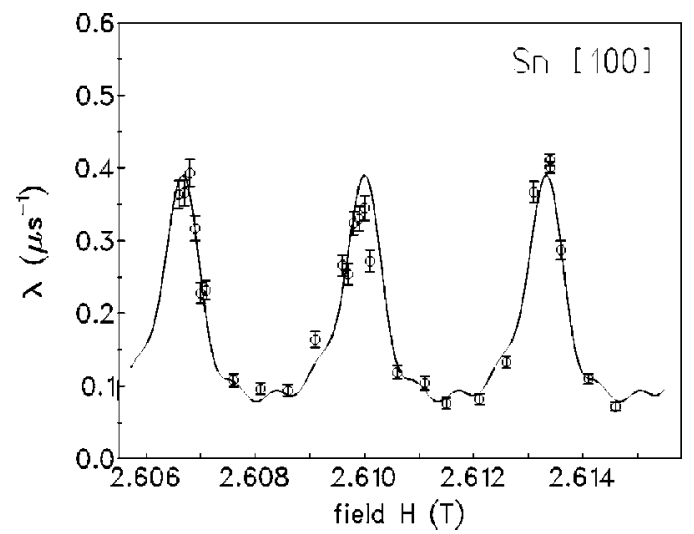

FIG. 3. Periodicity reappearing in the damping rate $\lambda(H)$ near $H=2.6 \mathrm{~T}$, related to the dHvA mode $\pi_{2}^{1}$. The expected period is $\Delta H=H^{2} / F\left(\pi_{2}^{1}\right)=32.8 \pm 0.4 \mathrm{G}$ (Ref. 19), the solid curve is a fit by a finite Fourier series (up to the 4th harmonics) with $\Delta H=33.3$ $\pm 0.005 \mathrm{G}$. The peak values $\lambda \approx 0.4 \mu \mathrm{s}^{-1}$ lead to the estimate $\Delta B \approx 9$ G (see Fig. 4). 


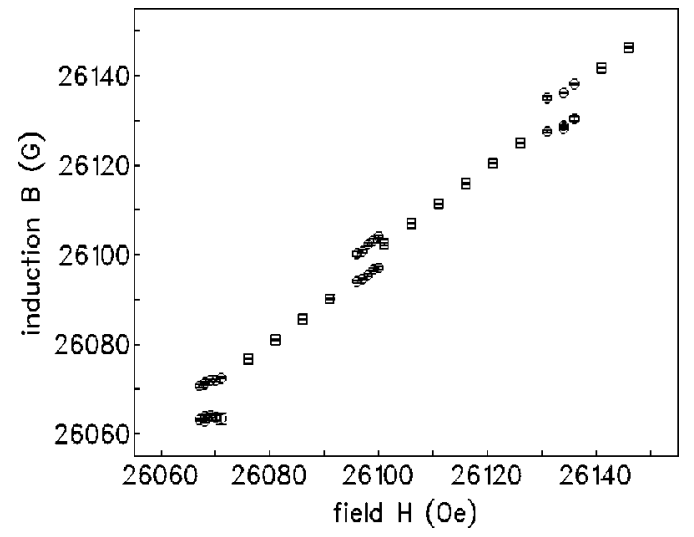

FIG. 4. Induction $B$ as a function of the applied field $H$ for the same field range as in Fig. 3. The two $B$ branches indicate sections with Condon domains, with a splitting of $\Delta B \approx 8 \mathrm{G}$. The nonideal shape of the plateaus is discussed in the text.

the maxima shifted upwards with increasing $m_{i}^{*} \cdot{ }^{2}$ As Figs. 1 and 3 show, for $H \leqslant 1.41 \mathrm{~T}$ and $H \geqslant 2.6 \mathrm{~T}$ one has the dominating oscillations $\tau_{2}^{1}$ and $\pi_{2}^{1}$, respectively. However, Eq. (1) is the general condition for a Condon instability, no matter whether this results from a single quantum oscillation or from a number of superposed modes. In the present case, as the increasing $H$ reaches the range $1.41<H<2.6 \mathrm{~T}$, the already decreasing amplitude for $\tau_{2}^{1}$ and the still growing one for $\pi_{2}^{1}$ become comparable and, at some $B$ values, the superposition of the two modes $\chi(B)=\Sigma_{i=\tau_{2}^{1}, \pi_{2}^{1}} \chi_{a}^{(i)} \cos \left(2 \pi F_{i} / B\right.$ $\left.+\phi_{i}\right)$ satisfies $\chi>1 / 4 \pi$. The irregular $\lambda(H)$ sequence observed at $H=2 \mathrm{~T}$ is due to the incommensurate frequencies, implying no periodicity for the position or length of the domain sections.

The maximum $\lambda \approx 0.4 \mu \mathrm{s}^{-1}$ in Fig. 3, if due to a doublet, gives the estimate $\Delta B \approx 2 \lambda / \gamma_{\mu} \leqslant 9 \mathrm{G}$, already resolvable for sufficiently narrow component lines. Although a splitting in frequency space was visible only for the top value at $H$ $=26067$ Oe, the systematic variation of the lineshape indicates indeed the presence of two components, with intensities "flowing" from the lower (diamagnetic) line to the higher (paramagnetic) one as $H$ increases.

The doublet structure is quantitatively confirmed by a standard analysis of the observed time series of the $\mu^{+}$polarization $P(t)$, by use of the fit function ${ }^{11}$

$$
P(t ; H)=\sum_{j=1,2} a_{j}(H) e^{-\lambda^{(j)}(H) t} \cos \left[\gamma_{\mu} B_{j}(H) t+\psi\right],
$$

with parameters $a_{j}, \lambda^{(j)}, B_{j}, \psi$. For a uniform state one expects to find $B_{1}(H)=B_{2}(H)$, while in the presence of Condon domains two distinct fields $B_{1}, B_{2}$ should result, both staying constant along the domain section of the dHvA cycle, whereas $a_{1}(H), a_{2}(H)$ should vary linearly, with the sum $a_{1}+a_{2}$ independent of $H$. The parameters were allowed to vary freely, except for the plausible restriction $\lambda^{(1)}$ $=\lambda^{(2)}$ for the doublet components.

The result of the fit for $B(H)$ is seen in Fig. 4, showing sections with two branches of $B$ separated by $\Delta B \approx 8 \mathrm{G}$ in each dHvA cycle. The approximately flat $B_{1}, B_{2}$ branches in the first and third cycles are characteristic to the domain state, though there are no "plateaus" in the middle domain

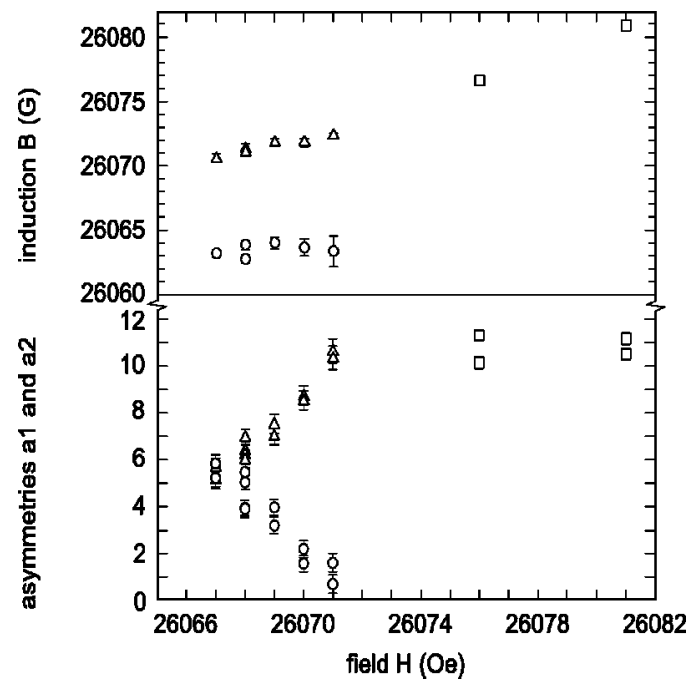

FIG. 5. Asymmetries ( $\propto$ volume fractions) $a_{1}(\bigcirc)$ and $a_{2}(\triangle)$ for the dia- and paramagnetic domains, respectively, in the first Condon domain section of Fig. 4, showing the predicted linear behavior. For clarity, part of the $B(H)$ curve is redrawn with the corresponding symbols for the two branches, the mark $\square$ stands for the uniform phase. The pairs of points for $a_{i}$ with the same symbol are data from two different detectors mounted at opposite sides of the sample.

section. The reason for this is not clear. An incomplete domain formation in some cycles may occur because of the smallness of the gain in free energy (scaled by $4 \pi \chi_{a}$ ) that drives the transformation, ${ }^{2}$ or due to the narrowness of the domain section $\delta H=n \Delta B \quad(n \approx 0.95$ is the demagnetizing coefficient for this sample). Also, $\Delta B$ here is still near to the resolution limit, and the statistical uncertainties in the data may lead to large distortions in the fitted shape of the $B(H)$ branches.

The $\mu^{+}$asymmetries (amplitudes) $a_{i}(H)$ of the doublet components are plotted in Fig. 5 for the first domain section of Fig. 4. The linear variations of $a_{1}$ and $a_{2}$ (for the dia- and paramagnetic components, respectively) with decreasing $H$ show the readjustment of the domain volumes predicted by the "Maxwell construction" for $\bar{B}=H$. For $H>26070$ Oe the crystal is uniform and the asymmetry is constant.

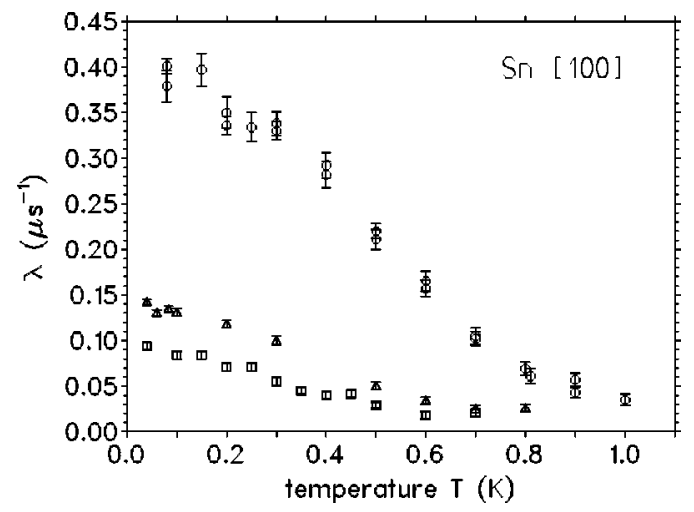

FIG. 6. Damping rate $\lambda$ vs temperature at different applied fields: $B=1 \mathrm{~T}(\square)$ and $1.4 \mathrm{~T}(\triangle)$ for mode $\tau_{2}^{1}$, and 2.6 $\mathrm{T}(\bigcirc)$ for mode $\pi_{2}^{1}$. Domains are absent above the critical temperature $T_{0}$, below $T_{0}$ the field splitting $\Delta B \propto \lambda(T)$ increases with decreasing temperature. 
With the increase of temperature the dHvA amplitude and thereby the splitting $\Delta B$ should, according to Eq. (2), monotonically decrease and $\Delta B$ disappears at $T=T_{0}$ determined by $4 \pi \chi_{a}^{(i)}\left(T_{0}\right)=1$. Figure 6 shows this predicted behavior of $\lambda(T) \propto \Delta B(T)$ for the two modes, at fields corresponding to equal volumes of dia- and paramagnetic regions. For $T$ $>T_{0}$ one has $\lambda \approx \lambda_{0}$, where $\lambda_{0}$ is characteristic for the uniform state of the sample and does not vary at this temperature range.

In conclusion, evidence for dia- and paramagnetic domains in white tin has been obtained. Even for the relatively low fields of $1-2.6 \mathrm{~T}$, the topology of the Fermi surface provides two domain generating $\mathrm{dHvA}$ oscillations for $\boldsymbol{H} \|[100]$ : mode $\tau_{2}^{1}$ is dominant for $H \leqslant 1.4 \mathrm{~T}$ and mode $\pi_{2}^{1}$ near $H=2.6 \mathrm{~T}$. In the intermediate field range domain formation is driven by the superposition of the two modes of comparable amplitudes, resulting in no periodicity in the sequence of domain states. The field splitting $\Delta B=4 \pi\left(M_{p}\right.$ $\left.-M_{d}\right) \approx 8 \mathrm{G}$ due to the opposite domain magnetizations $M_{d}, M_{p}$ could, for the $\pi_{2}^{1}$ mode, be resolved in the $\mu \mathrm{SR}$ signal, with line intensities showing the predicted linear behavior. For the $\tau_{2}^{1}$ oscillation $\Delta B$ is smaller, but the doublet structure, unresolved in this case, is manifest in itself in the periodically arising sharp peaks of the line broadening $\lambda(B)$. For both "domain active" modes, $\lambda(T) \propto \Delta B(T)$ decreases monotonically with increasing temperature.

We are greatly indebted to V. F. Gantmakher for providing us with the tin crystal, and to the PSI staff members for the excellent muon beam. One of the authors (V.E.) is grateful for Grant No. 98-02-17142 of the Russian Fund for Fundamental Research.
${ }^{1}$ J.H. Condon, Phys. Rev. 145, 526 (1966).

${ }^{2}$ D. Shoenberg, Magnetic Oscillations in Metals (Cambridge University Press, Cambridge, 1984).

${ }^{3}$ Ya. Blanter, M.I. Kaganov, and D.V. Posvyanskii, Phys. Usp. 38, 203 (1995).

${ }^{4}$ A.A. Varlamov, V.S. Egorov, and A.V. Pantsulaya, Adv. Phys. 38, 469 (1989).

${ }^{5}$ I.D. Vagner, T. Maniv, and E. Ehrenfreund, Phys. Rev. Lett. 51, 1700 (1983).

${ }^{6}$ R.S. Markiewicz, Phys. Rev. B 34, 4172 (1980).

${ }^{7}$ A. Gordon, M.A. Itskovsky, and P. Wyder, Phys. Rev. B 55, 812 (1997).

${ }^{8}$ A. Gordon, M.A. Itskovsky, I.D. Vagner, and P. Wyder, Phys. Rev. Lett. 81, 2787 (1998).

${ }^{9}$ J.H. Condon and R.E. Walstedt, Phys. Rev. Lett. 21, 612 (1968).

${ }^{10}$ G. Solt, C. Baines, V.S. Egorov, D. Herlach, E. Krasnoperov, and U. Zimmermann, Phys. Rev. Lett. 76, 2575 (1996).

${ }^{11}$ G. Solt, C. Baines, V.S. Egorov, D. Herlach, and U. Zimmermann, Phys. Rev. B 59, 6834 (1999).

${ }^{12}$ A.B. Pippard, in Electrons at the Fermi Surface, edited by M. Springford (Cambridge University Press, Cambridge, 1980).

${ }^{13}$ V.F. Gantmakher, Zh. Éksp. Teor. Fiz. 44, 811 (1963) [Sov. Phys. JETP 17, 549 (1963); 46, 2028 (1964) [19, 1366
(1964)].

${ }^{14}$ M.D. Stafleu and A.R. de Vroomen, Phys. Status Solidi 23, 676 (1967); 23, 683 (1967).

${ }^{15}$ J.E. Craven, Phys. Rev. 182, 694 (1969).

${ }^{16}$ W.A. Roger and S.B. Woods, J. Phys. F: Met. Phys. 6, 2289 (1976).

${ }^{17}$ R.W. Vaughan, D.D. Elleman, and D.G. McDonald, J. Phys. Chem. Solids 31, 117 (1970).

${ }^{18}$ J.M. Deacon and L. Mackinnon, J. Phys. F: Met. Phys. 3, 2082 (1973).

${ }^{19}$ V.M. Pudalov and M.S. Khaikin, Zh. Éksp. Teor. Fiz. 67, 2260 (1974) [Sov. Phys. JETP 40, 1121 (1974)].

${ }^{20}$ A. Schenck, Muon Spin Rotation Spectroscopy (Hilger, Bristol, 1986).

${ }^{21}$ R. Abela, A. Amato, C. Baines, X. Donath, R. Erne, D.C. George, D. Herlach, G. Irminger, I.D. Reid, D. Renker, G. Solt, D. Suhi, M. Werner, and U. Zimmermann, Hyperfine Interact. 120-121, 575 (1999).

${ }^{22}$ G. Solt, C. Baines, V.S. Egorov, D. Herlach, and U. Zimmermann, Proceedings of the 44th Annual Conference on Magnetism and Magnetic Materials, San Jose, CA, 1999 [J. Appl. Phys. 87, 7144 (2000)]. 